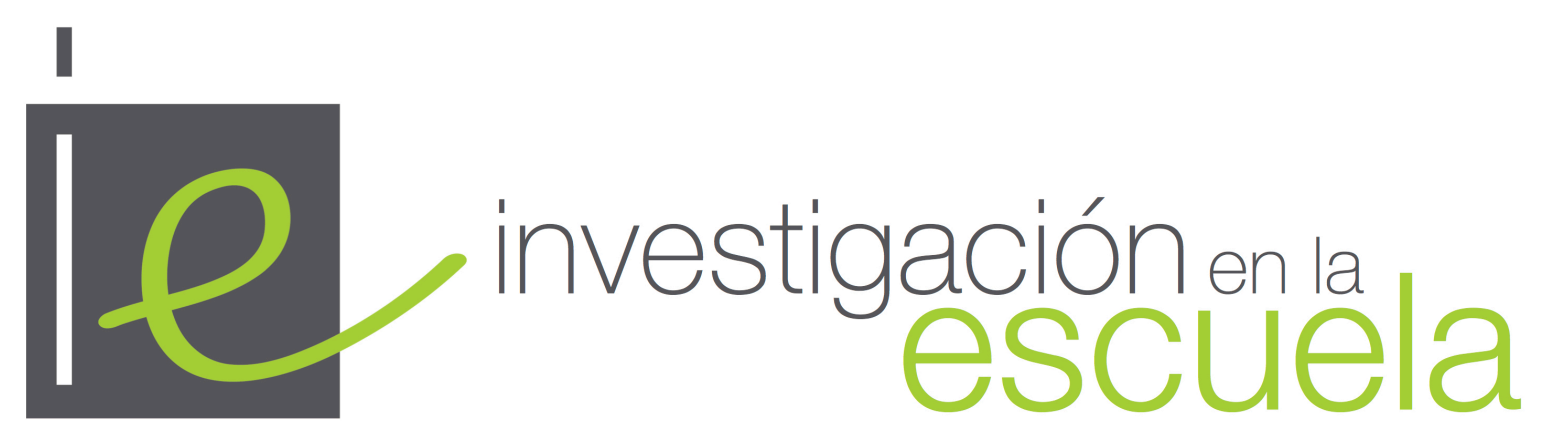

Revista internacional de investigación e innovación educativa

Número 100

30 de abril de 2020

ISSN 2443-9991

\title{
Educación para el Desarrollo Sostenible: del proyecto cosmopolita a la ciberconvivencia
}

\author{
Rosario Ortega Ruíz. \\ Universidad de Córdoba \\ España
}

Citación: Ortega Ruíz, R. (2020). Educación para el Desarrollo Sostenible: del proyecto cosmopolita a la ciberconvivencia. Investigación en la Escuela, 100, 11-22. DOI:

https://dx.doi.org/10.12795/IE.2020.1100.02

Resumen: El artículo presenta una reflexión sobre el papel de la educación para afrontar los Objetivos del Desarrollo Sostenible que sugiere la Agenda 2030. Desde el importante movimiento cultural de finales del siglo XX que ya se preocupaba por los grandes retos de la educación y la cultura para afrontar los profundos cambios que la globalización imponía, analizamos la interrupción del proyecto cosmopolita y la necesidad de retomarlo de nuevo, incorporando el reto de la convivencia y la cyberconvivencia. Las investigaciones sobre acoso y ciberacoso nos han llevado a poner en evidencia la importancia de profundizar en la atención a la sensibilidad moral y la competencia social para lograr que escolares y jóvenes tomen conciencia de los valores de prosocialidad y altruismo para construirse un criterio capaz de asumir los objetivos para el desarrollo sostenible.

Palabras clave: "Sostenibilidad"; "cosmopolita"; "ciberconvivencia"; "acoso escolar"; "prosocialidad".

\section{Education for Sustainable Development: from the cosmopolitan project to cyber coexistence}




\begin{abstract}
The article presents a reflection on the role of education in the face of the Sustainable Development Goals as defined in the 2030 Agenda, through Education for Sustainable Development. Starting with a reflection on the challenges that globalization brought to culture and education on the late twentieth century, we analyse how the cosmopolitan project was interrupted and reflect on the need to re-ignite it, incorporating the challenges that "cyber-convivencia" brings in. Research on bullying and cyberbullying has led us to highlight the importance of deepening our attention to moral sensitivity and social competence, to make schoolchildren and young people aware of the value of pro-sociality and altruism so that they can develop criteria to incorporate the Sustainable Goals.
\end{abstract}

Key words: "Sustainability"; "cosmopolitan"; "cyber-convivencia"; "bullying"; "prosociality".

\title{
Éducation au développement durable: du projet cosmopolite à la cyber-coexistence
}

Resumè: Cet article présente une réflexion sur le rôle de l'éducation pour faire face aux Objectifs de Développement Durable proposés par le Agenda 2030. A partir de l'important mouvement culturel de la fin du XXe siècle qui était déjà concerné par les grands défis de l'éducation et de la culture pour faire face aux profonds changements que la mondialisation a imposés, nous analysons l'interruption du projet cosmopolite et la nécessité de le reprendre, en incorporant le défi de la "cyberconvivencia". La recherche sur l'intimidation et la cyberintimidation nous amène à souligner l'importance d'approfondir l'attention sur la sensibilité morale et à la compétence sociale pour garantir que les écoliers et les jeunes puissent construire un critère capable d'assumer les objectifs de développement durable.

Mots-clés: "Durabilité"; "cosmopolite”; “cyberconvivencia”; "intimidation"; "prosocialité".

\section{Introducción}

Investigación en la Escuela -IE- es algo más que el nombre de una revista de investigación y práctica educativa. Investigación en la Escuela es la feliz expresión de un activista movimiento de profesores y profesoras, docentes, estudiantes de Magisterio y educadores en general que buscaban, desde los inquietos años setenta y ochenta del siglo pasado, caminos por donde transitar sus inquietudes intelectuales y pedagógicas (García-Pérez, 2016). Han pasado más de treinta años y es tiempo de reflexionar sobre el impacto que la teoría tiene sobre la práctica educativa, al tiempo que analizar el que la práctica educativa ejerce sobre la cultura y la sociedad en general. Más allá de la teoría socio-política y la sociología de la educación, nos acercaremos al tema a través de la psicología de la educación. La psicología de la educación y el desarrollo es una disciplina que sin dejar de ser parte de las ciencias psicológicas forma parte, por derecho propio, de las ciencias de la educación; los procesos psicológicos que se activan en los escenarios y en las funciones de aprendizaje y enseñanza son parte final de una cadena de procesos que articula la educación como práctica y ésta incide, a su vez y por distintos mecanismos, en la cultura y el desarrollo de las personas y las comunidades. En lo que sigue se presenta una reflexión teórica sobre este tema bajo cuatro epígrafes que articulan un discurso que pretende ser crítico y positivo, con los siguientes títulos: a) mundialización y crisis cultural: el proyecto cosmopolita; b) la Agenda 2030 y los Objetivos para el Desarrollo Sostenible; c) convivencia y ciberconvivencia: escenarios de la experiencia educativa; y d) Educación para el Desarrollo Sostenible: la prosocialidad como motor. 


\section{Mundialización y crisis cultural: el proyecto cosmopolita}

En los últimos años del siglo XX hervía entre los y las intelectuales de la época el impacto de la globalización económica, las entonces denominadas nuevas tecnologías -TIC- y en general la mundialización financiera y su efecto devastador en la cultura. Ello fue estímulo para repensar la educación en su teoría y en su práctica. Fueron años ricos en ideas sobre el papel de la educación y su posible función de corrección de lo que se pensaba que era el riesgo de la deshumanización. Fueron años en los que las ciencias sociales empezaron a brillar en innovación e investigación. Años de finales del siglo pasado y de comienzos del siglo XXI que dieron a las ciencias sociales en general, y a la educación en concreto, un escenario para la reflexión y el análisis que me temo que se ha perdido en la actualidad, o que corre el riesgo de perderse por su escasa presencia en los medios expertos y populares a los que accedemos. Parecía que los paradigmas clásicos de las ciencias admitían un nuevo paradigma abierto y global para las ciencias sociales que, asumiendo el rigor del control metodológico, no ignorara que el pensamiento científico debe abrirse a los problemas humanos tal y como se presentan en un mundo global y tecnológico. Morin (2004) afrontó con rigurosa sensibilidad una reformulación del asunto, estableciendo una evolución de la epistemología científica en cinco grandes modelos, que van del empírico-racional al modelo de la complejidad, reasumiendo los modelos genético, evolutivo e historicista. En definitiva, lo que hace Morin es establecer un camino historicista para llegar a la complejidad como paradigma, con independencia de que sean solo razones históricas las que aporten sus argumentos. De aquellos fuegos intelectuales queda un interesante rescoldo: un mundo global demanda un análisis complejo, y las ciencias sociales tienen que dar una respuesta comprensiva. Así se comenzó a tratar de interpretar y comprender qué clase de cultura sobreviviría a la globalización financiera y a la impiedad de la injusta distribución de la riqueza y el poder en la sociedad de la información y la comunicación. Algunos fueron muy pesimistas. Habermas (2001) tituló su emblemático artículo sobre el impacto de la globalización en la cultura de forma brutal: "La alienación cultural, o el valle de lágrimas de la globalización" y Álvarez Munárriz (2001), más prudente y precavido, nos alertaba de la necesidad de participar en el debate social de la mundialización porque era algo que nos afectaba directamente a todos; algo que incidiría en el futuro de nuestras vidas. Pero no cabe duda de que quien más claramente intervino en el apasionante debate sobre globalización, cultura y educación fue el profesor Manuel Castells (1996), que supo señalar que sería la rapidez y el dominio de la comunicación que circula en la Red por excelencia (Internet) y en general la digitalización de nuestras vidas, lo que aportaría los grandes parámetros de la cultura y la educación, lógicamente dependiendo de cómo los gobiernos y en general la gobernanza actuaran al respecto.

El rico debate transcendía la sociología y la economía e interesaba a todos los que deseábamos encontrar un camino para la mejora de la educación, más allá de lo que en los años setenta y ochenta del siglo pasado habían intentado los caóticos movimientos de innovación pedagógica, ligados a alternativas ideológicas aún de marcado carácter marxista (Apple, 1991). Se abrió para la ciencia social en general, y para la educación en concreto, un escenario donde la reflexión y el análisis parecía que podían articular globalización y cambio cultural. Pesimistas y optimistas animaban la discusión. A los pesimistas se les oponían los optimistas e incluso los arrojados e imprudentes. Instituciones como la London School of Economic (hoy parte de la Universidad de Londres, pero ya en aquel momento vanguardia del saber socio-económico y político) se convirtieron en hervideros de ideas, iniciativas y a veces aventuras, destinadas a analizar, comprender y diseminar el importante papel que la cultura y la socialización tendrían en un mundo ya global en la economía y en las finanzas, que amenazaba serlo también en la política y el poder de los gobiernos nacionales. Al desparpajo con el que Giddens (1991) había calificado la educación como una práctica social que se reinterpretaría a la luz del examen que la misma práctica haría, le 
siguió el entusiasmo con el que Held y McGrew (2000) señalaban la oportunidad de que la cultura y la socialización podrían verse afectadas por los benéficos de la educación. Ello tendría un gran impacto en la mejora de las condiciones de vida, el reparto de la riqueza y en general, una nueva y esperanzadora etapa en la cual la mundialización mejoraría las condiciones de vida de los seres humanos. Este optimismo encontró replica en los trabajos de Spring (2001), en los que se ponía de relieve que había más riesgos que alegrías en confiar en la educación como motor de cambio. La falta de inversión terminaría defraudando las esperanzas que se estaban poniendo en la potencialidad, tanto de la tecnología como de la transformación, que la formación educativa podría tener en la vida de las personas. El debate era controvertido, pero al menos había debate, al menos teníamos tema y caminos para transitar en la búsqueda de un mundo mejor, ya que era evidente que los grandes cambios tecnológico-financieros estaban transformando el mundo.

Bajo la inspiración del análisis socio-político y epistemológico y tomando la etiqueta de Held y McGrew (2000), nos acercamos a un reto educativo: el proyecto cosmopolita. El proyecto cosmopolita, visto desde la psicología (Ortega-Ruiz, 2005; 2009), focalizó sus objetivos en lograr que los escolares llegaran a construirse como seres psicológicamente equilibrados y seguros de sí mismos, al tiempo que les mostró el reto de conocer el mundo tal y como es, en su universalidad y mundialización. En aquellos años (Ortega-Ruiz y Martín-Ortega, 2004) escribíamos que si un ciudadano/a cosmopolita es una persona que obedece intelectualmente a un sistema democrático y capaz de establecer diálogos con tradiciones, discursos y formas culturales diversas, la educación debería tener como finalidad que los niños y las niñas comprendieran el significado de la democracia como el escenario compartido donde ser un buen conciudadano y ciudadana, que respeta la diversidad de culturas y la pluralidad de intereses y discursos. En mi opinión, el proyecto cosmopolita, con su dosis de idealismo y sus fundamentos en el pragmatismo, aún está vigente porque, o tenemos una educación que invierta en valores para la democracia y el entrenamiento de una mente creativa e innovadora, o no dispondremos de herramientas para construir el futuro.

El proyecto cosmopolita tenía mucho de utopía, pero también de realismo. Igual que la IE, que tiene mucho de ingenuidad y mucho de ambición. Cuando se enuncian los principios de la IE no estamos intentando tener científicos en pequeñito, sino proponiendo que la práctica educativa estimule la curiosidad, la motivación por el saber, la inteligencia creativa y la innovación en la actividad de docentes y escolares (Cañal y Porlán, 1987). Es decir, la IE se formula como herramienta útil para estimular el proceso de aprendizaje y enseñanza, buscando mayores niveles de calidad educativa.

Un día, acontecimientos de infausta memoria relacionados con el terrorismo suicida y la corrupción política y económica nos dejaron sin debate. El debate social y cultural fue sustituido de nuevo por la guerra, la religión y particularmente la miseria que asolaba la vida de los ciudadanos y ciudadanas, especialmente los más vulnerables, que pagaron la factura de los platos que no habían roto. El discurso de la guerra, la exclusión social, la inmigración obligada por el dolor y la crueldad ideológica o financiera, a quienes más atacó, además de a las mujeres, fue a la infancia y a aquellos que dependen de los recursos sociales para educarse y sobrevivir.

Sin debate crítico, achicada la motivación de las grandes ideas, nos recluimos cada quien en lo suyo, huérfanos de la dimensión crítica y universal del tema que nos afecta: la posibilidad de que la educación sea el impulso sabio y valiente para construir un mundo mejor. Quizás, como diría Giddens (1991), la práctica educativa se olvidó de su dimensión universal y civilizatoria para sobrevivir con los recursos menguantes y las acostumbradas rutinas de calidad y alcance disminuido. Han pasado veinte años y el interés por el análisis global y universalista del papel de la educación y la cultura en la sociedad no parece estar en la agenda de las ciencias sociales. 


\section{La Agenda 2030 y los Objetivos para el Desarrollo Sostenible}

Hoy parece que es el riesgo medioambiental lo que nos convoca de nuevo a un debate universal. Revisando los diecisiete Objetivos para el Desarrollo Sostenible -ODS- que establece la Agenda 2030 de las Naciones Unidas, quizás tengamos la oportunidad de volver a pensar en la importancia de la educación y su papel como motor de un mundo mejor, y quizás reeditar el proyecto cosmopolita.

Riesgos que ponen en evidencia la posibilidad de que se deteriore el planeta Tierra hasta niveles irreversibles por el impacto que el ser humano y sus depredadoras formas de vida está ejerciendo sobre él. El uso abusivo del consumo por parte de unos, y la permanencia en la indigencia, la indignidad y el subdesarrollo de otros, en un mundo ya interconectado, que globaliza desde el intercambio de información hasta las pandemias, exigen volver a la discusión sobre el papel de la educación y la cultura. Hoy tenemos sólidos motivos para volver a pensar en la educación como un proyecto cultural universal.

La emergencia climática se ha convertido en el gran reto. En un mundo totalmente globalizado, no está claro cómo podemos afrontar, incluso si llegaremos a tiempo de detener las catástrofes y desastres naturales, la compleja tarea de mantener los actuales niveles de civilización. El reto de la sostenibilidad y los ODS nos están indicando hacia dónde hay que girar, hacia dónde habría que mirar. De entre ellos, el objetivo cuatro formula la necesidad de "garantizar una educación inclusiva, equitativa y de calidad y promover oportunidades de aprendizaje durante toda la vida para todos”. Pero es el conjunto de diecisiete objetivos lo que puede iluminar el análisis.

Aunque parece evidente que desde las actuales condiciones de la cultura, instituciones y socialización quizás no lo consigamos para nuestro ciclo vital, los ODS se deben convertir en herramientas para la educación de las nuevas generaciones. Es necesario que los ciudadanos y ciudadanas del futuro estén preparados para actuar con celeridad, inteligencia y precisión para cambiar su propio modo de vida antes de que el cambio climático convierta nuestro ecosistema en un escenario imposible para sobrevivir y mantener el Planeta. El importante papel que están teniendo los movimientos juveniles de alerta ante la emergencia climática, como el denominado Friday for Future, subtitulado School Strike for Climate, que encabeza la joven Greta Thunberg, debe alertarnos a parar y dedicar una reflexión sobre qué estamos haciendo y qué es lo que debemos hacer en educación si la tarea es global, universal y urgente.

El debate queda abierto. Lo han abierto los jóvenes, nuestro alumnado adolescente y juvenil que con lógico enfado nos reprochan la pasividad que estamos exhibiendo ante la emergencia climática. Jóvenes que nos exigen que no les dejemos en herencia un mundo devastado, sino un mundo en el que ellos puedan desarrollar su propia vida y legarlo a sus hijos e hijas en mejores condiciones que lo recibieron. Los ODS nos retan a que vayamos poniendo en claro lo que pensamos que la educación, como práctica universal, puede hacer para contribuir a la construcción de un mundo sostenible y habitable.

Retomemos pues el debate que quedó silenciado por la corrupción y la barbarie. Después de 20 años las preguntas siguen abiertas y hay cuestiones en todos los órdenes: ¿es la educación una herramienta necesaria para modificar la cultura ?, ¿qué papel tiene la educación en el desarrollo de las personas y las comunidades, si lo que buscamos es un mundo mejor?, ¿la inteligencia digital estimula el aprendizaje y el desarrollo de los individuos, o por el contrario cierra puertas a quienes no ajustan sus competencias básicas a las exigencias de un mundo digitalizado?, ¿estamos preparados para asumir un modo de vida que parece imponer a muchos estar conectados mediante un dispositivo digital once horas al día?, ¿qué tiempo nos queda para realizar la socialización?, ¿qué parte de la socialización imprescindible nos perdemos diariamente?, ¿nuestra capacidad de procesar información es compatible con la comprensión del mundo?, ¿el dominio de recursos tecnológicos 
permite la transformación de la mente sin destruir el necesario equilibrio emocional y el bienestar personal y social? La tecnología digital, la comunicación instantánea y el poder de las redes sociales sin duda agilizan la información, pero ¿está fragmentando la comunicación?, ¿estamos más solos frente a la fría pantalla?, ¿qué impacto tendrá la soledad digital en la construcción del equilibrio emocional?, ¿cómo se reestructurará la formación en actitudes y valores si se deteriora el vínculo afectivo necesario? Si la investigación empieza por la formulación de una o varias preguntas, en mi opinión, la innovación educativa tiene una enorme tarea científica por delante.

Sin pretender responder a todas las preguntas y advirtiendo de que no se trata de cuestiones técnicas sino de preguntas para la investigación y la reflexión, es claro que lo que necesitamos repensar son las metas y su coherencia con las prácticas educativas. Necesitamos una enseñanza y un aprendizaje más instrumental, que desarrollen competencias y habilidades, pero también una educación más extensa, más profunda, más crítica, que incidan en las actitudes, los valores y la conducta; una educación que forme en la mente de los escolares sólidos cimientos éticos y prácticos; una educación que estimule la inteligencia para llegar lo más lejos posible en la capacidad cognitiva para comprender y actuar en el complejo mundo en el que vivimos, sin destruirlo. ¿Es ello posible en un mundo en el que la tecnología parece haber suplantado todos los retos y esperanzas del proyecto cosmopolita? En el escenario están las crisis económicas que paralizan el motor de unos sistemas educativos concebidos por ciertos gobernantes más como un negocio ideológico que como un servicio al progreso de toda la comunidad. Pero también están los fenómenos más específicamente sociales de la mundialización, como las migraciones que guerras y catástrofes medioambientales producen: hay que atender a víctimas, expatriados y refugiados; el gasto armamentístico, la corrupción económica y los malos gobernantes ocupan nuestra atención; ‘alguien se ocupa de la educación?, ¿se enuncian los retos educativos de forma clara en la gobernanza global y en las leyes y normas educativas nacionales y regionales?, ¿'somos conscientes de que la educación modula las formas de pensamiento, pero también las actitudes, sentimientos y valores y así impacta a la cultura y la sociedad?, ¿cómo habrá de configurarse la mente humana para sustituir la conducta depredadora por la solidaria? Disciplinadamente elegimos para el análisis el Programa de Acción Mundial para la Sostenibilidad en la Agenda 2030 y sus diecisiete objetivos, de los cuales se deriva la Educación para el Desarrollo Sostenible -EDS- que debe guiarnos.

La EDS arranca de los dos parámetros que determinan la práctica educativa: la socialización y la institucionalización. La educación es parte fundamental del más amplio y complejo proceso socializador, que espera incidir de forma significativa en las mentes, las actitudes y la conducta humana mediante el aprendizaje y la conformación de comunidades. Socialización e institucionalización son constructos que se concretan en teoría y práctica educativa. Si la EDS es el programa universal mínimo, la práctica tiene que mirar a las experiencias socializadoras que las instituciones educativas están proporcionando a los y las escolares en su vida diaria. Hay que relajarse un poco respecto de lo que puede dar de sí el currículum, porque no se trata tanto de lo que se enseña, sino de cómo se enseña y de cómo se experimenta la vida mientras se aprende. Hay que pensar un poco más en las consecuencias de vivir, o no, de forma plena en contacto con el conocimiento y las relaciones interpersonales positivas y afectivamente satisfactorias, que hagan posible ir construyéndose una identidad personal y una agencia autónoma moralmente responsable. Es decir, la convivencia.

\section{Convivencia y ciberconvivencia: escenarios de la experiencia educativa}

La convivencia escolar es el conjunto de sistemas de relaciones sociales e interpersonales vinculados a la actividad educativa -enseñanza y aprendizaje- que sostiene y da sentido a la vida 
social en las aulas y en los centros escolares (Ortega-Ruiz, 2015). En una investigación en la que se validaba el constructo convivencia desde el punto de vista de los escolares y los docentes (Del Rey, Casas y Ortega-Ruiz, 2017) encontramos que la mejor aproximación al mismo incluía tres grandes dimensiones: a) criterios psicoeducativos relacionados con la actitud y estilo relacional de los docentes (tearchers' attitude); b) criterios relacionados con el bienestar compartido (wellbeing) y el conjunto de normas que regulan la vida en común (school climate), particularmente las normas explícitas e implícitas que sustentan las relaciones interpersonales de los escolares entre sí; y c) criterios relacionados con los fenómenos que perturban el bienestar, deterioran el clima social y alimentan el desapego y la desconfianza de los escolares hacia la institución y las relaciones comunes (interpersonal relationship). Este estudio, realizado con una amplia muestra representativa de escolares andaluces, confirmó algo que veníamos suponiendo: que en el mundo de los iguales está la clave de la sociabilidad y que el ajuste y equilibrio afectivo entre los escolares es determinante. Fenómenos como la intimidación, la exclusión, los malos tratos, el abuso de poder, el acoso y hostigamiento, así como la agresión verbal y física perturban el bienestar y la seguridad necesarios para aprovechar la enseñanza y realizar la función del aprendizaje. Pero descubrimos algo que, aunque siempre lo sospechábamos, no habíamos incluido hasta ese momento en nuestros trabajos previos: que es la calidad de la actitud y la conducta del docente, la dimensión que tiene más peso en la percepción que los escolares tienen sobre la calidad y seguridad que su escuela les ofrece.

La convivencia es un constructo que forma parte de la cultura escolar desde distintos puntos de vista y que es usada para distintos propósitos pedagógicos. Aquí nos interesa el concepto convivencia en lo que tiene de dimensión compleja para la socialización y la institucionalización de la práctica educativa. Se necesita un buen ecosistema social -una buena convivencia- para que la escuela realice la función de socializar y lograr que los escolares comprendan, mediante el respeto a lo que pasa en su escuela, el valor del bien común compartido. Se necesita que la convivencia sea percibida por los escolares como el marco institucional que comparten todos los protagonistas y en el que acontecen todos los asuntos importantes de la vida en la escuela. Una buena convivencia está compuesta del entramado de afectos, comunicación y actividad compartida, que se percibe como la naturaleza misma de la vida escolar.

La convivencia impacta el proceso cognitivo y afectivo modulando la construcción progresiva de la personalidad de los escolares, que deben ir aumentando en competencia social, pero también en autonomía e independencia. En el entramado de relaciones interpersonales que se crea en las aulas, la empatía se transforma en solidaridad y el patrón agresivo-defensivo en competencia e inteligencia social. La buena convivencia alimenta la autoestima y sostiene la agencia, logrando en los miembros del ecosistema un equilibro sano y gratificante entre sus propios intereses y la progresiva adaptación a costumbres y normas comunes. En el entramado de la convivencia con los iguales se modulan afectos y emociones, suavizando los impulsos disruptivos que la frustración o la dificultad del aprendizaje producen en escolares y docentes.

La convivencia modula los conflictos, transformando en sociales y adaptativas las respuestas a fenómenos indeseados, contribuyendo a la sociabilidad, la competencia social y la toma cooperativa de decisiones. Solo así se adquiere el sentido de comunidad que será la base socializadora e institucional de lo que luego se identificará como ciudadanía. Solo así se experimenta la complejidad del bien común, la operatividad de la norma democrática y del papel de cada uno en la construcción de las normas que regulan los agrupamientos y las instituciones.

Ciertamente que la vida familiar y los patrones educativos de padres y madres ejercen sobre la personalidad social de los niños y las niñas un gran impacto (Gómez-Ortiz, Romera y OrtegaRuiz, 2016), pero la convivencia escolar es el escenario público que les permite entrenarse, sin la benevolencia que aportan los afectos íntimos, en la tarea de ser aprendices de ciudadanía. Hay que prestar atención a la experiencia de la vida en común, hacia la forma en que los escolares aprenden a 
negociar la cooperación y el conflicto, así como al proceso de distinguir, con autonomía y coherencia, lo que es aceptable y lo que no lo es por injusto o injustificado. Un buen ejemplo de esto lo tenemos al estudiar el fenómeno del acoso escolar (bullying). En el bullying (Ortega-Ruiz, 2010) todos los escolares se enfrentan, en vivo y en directo, ante la injusticia y el abuso de poder que uno o varios escolares ejercen sobre otro que normalmente es más vulnerable y no encuentra cómo defenderse. Todos los escolares conocen bien cómo funciona el bullying y cada quien juega un papel en ese perturbador fenómeno social de aulas y centros. La forma en que cada uno actúa ante la intimidación y el abuso de poder da la medida de cómo está evolucionando su sensibilidad y su criterio moral.

Pero hoy, como consecuencia del uso generalizado de los dispositivos digitales, el bullying se prolonga en el cyberbullying, y la convivencia en la ciberconvivencia (Ortega-Ruiz y Zych, 2016). Las redes sociales amplían las posibilidades de amistad, comunicación y el fluir creativo de iniciativas y proyectos comunes. Pero también en las redes sociales anida el abuso de poder, la intimidación, la exclusión social y en general la agresividad injustificada. La ciberagresión y la cibervictimización destruyen la convivencia y la ciberconvivencia, dañando los sentimientos y por ende la sensibilidad moral y el progreso socializador (Ortega-Ruiz, Del Rey y Casas, 2015). Más allá del valor de la información y la comunicación, los dispositivos digitales transforman el escenario donde es posible educar para la ciudadanía. Nos interesa el ciberespacio como prolongación de la vida en común en la que también es importante estimular la sensibilidad moral.

Solo un sistema de convivencia y ciberconvivencia escolar y juvenil que incorpore la sensibilidad moral para convertir la empatía en solidaridad creará en los escolares un pensamiento autónomo y crítico ante la irracionalidad de la injusticia, que es lo que necesitarán para tomar las decisiones comunes en un mundo global e interconectado que hay que hacer sostenible.

\section{Educación para el Desarrollo Sostenible: la prosocialidad como motor}

El proyecto cosmopolita nació del análisis socio-político y económico que el fenómeno de la globalización y la tecnologización de las comunicaciones implantaba en la sociedad. Pero el impacto de la tecnología de la información y la comunicación -TIC- no ha aportado, por sí mismo, el modelo de educación universalista y cosmopolita que se buscaba. Veinte años después, si hoy apostamos por una educación para el desarrollo sostenible, debemos seguir aspirando a que la escuela eduque a los niños y niñas, adolescentes y jóvenes, para convertirse en verdaderos ciudadanos y ciudadanas del mundo, capaces de lograr los ODS de la Agenda 2030 a los que nos hemos comprometido. Para ello no solo debemos atender a la experiencia compartida de la convivencia escolar, porque es ahí donde acontecen las experiencias interactivas significativas para la construcción de la personalidad y el criterio moral que están en la base del pensamiento crítico, sino que debemos atender también a la ciberconvivencia. Lo que ocurre en la ciberconvivencia también está impactando la mente, las actitudes, la sensibilidad moral y las conductas de los niños y niñas, adolescentes y jóvenes. Complejos procesos de ciberconducta nos retan a seguir investigando cómo funcionan las tensiones emocionales, el liderazgo en las redes y la necesidad de popularidad, o el seguidismo y la sumisión social, a comprender cómo emergen los códigos y normas implícitas y salientes en los escenarios de las redes sociales y las claves de la ciberconvivencia. Se trata de complejos hilos interpersonales de vinculación y filiación o de antagonismo y rechazo que los escolares actualizan más allá de las aulas y los pasillos del centro, y que son los que sostienen la vida en común de los iguales, con sus códigos y sus normas. El posible desajuste entre la identidad y la autoestima autopercibida y la imagen social y la identidad ejercida en la red social, el compulsivo deseo de destacar o el seguidismo y el gregarismo que se expresa al pulsar un "me gusta", o elegir un icono con el pulgar hacia arriba o hacia abajo en 
la red compartida, resultan importantes frecuentemente para la vida sentimental y el equilibrio social de los escolares.

La EDS exige un esfuerzo para integrar el mundo digital en el proyecto universalista de convertir a los escolares en ciudadanos y ciudadanas con sensibilidad moral y criterio ético, simplemente porque el escenario digital está investido, por los escolares, con los valores con los que construyen sus comunidades de convivencia. Simplemente porque la ciberconvivencia es una prolongación de la convivencia.

Si lo que buscamos con una EDS es conseguir sociedades que cuiden el planeta, solo lo conseguiremos si logramos educar individuos altruistas que estén moralmente contra la violencia injustificada y que desarrollen un pensamiento, actitud y ética prosocial. El planeta Tierra, la emergencia climática y la sostenibilidad de los parámetros civilizatorios no la haremos los que hoy escribimos en las revistas especializadas de educación, sino nuestros alumnos y alumnas, y la harán con lo que pueble sus mentes: inteligencia y conocimientos sobre el mundo, sus sistemas y sus procesos, pero sobre todo con valores éticos y sensibilidad moral hacia sus conciudadanos y hacia ellos mismos. La creativa hipótesis de Trivers (1971) que relacionaba la sobrevivencia de la especie humana con vínculos y afectos de sociabilidad entre los que, sin estar emparentados, se aprecian lo suficiente como para que emerjan entre ellos ideas altruistas, está llena de sentido. Es la prosocialidad entre personas no emparentadas, pero que construyen y respetan normas y proyectos que van más allá de sus propios intereses personales, lo que hace posible que se respete el bien común. Ello solo es posible si los individuos están equipados con ciertas habilidades cognitivas, como la capacidad para reconocer la sensibilidad moral mutua que se proyecta en sociedades e instituciones.

Ciertas emociones de amor y filiación, como la simpatía y la empatía, la confianza y la gratitud, tejen esa red afectiva común, pero también la vergüenza y la culpa son emociones morales que sostienen la sensibilidad ética. Ambas, las que nos acercan a los demás y las que controlan que no nos hagamos daño entre nosotros son las emociones requeridas para la construcción plena de un criterio moral que se sostenga en el tiempo, que dure en la memoria individual y solidifique una memoria compartida. Un criterio moral que no será, como se suponía en los años setenta y ochenta del siglo XX, un frío razonamiento lógico, sino una posición empática y solidaria hacia la necesidad de buscar acuerdos que garanticen beneficios comunes, evitando el egocentrismo intelectual y el egoísmo narcisista.

Sin una profunda y clara visión cognitiva y afectiva del criterio moral articulado a una ética universal no se garantiza que los habitantes de este Planeta estén en condiciones de afrontar conjuntamente la tarea de rectificar la emergencia climática y apostar por un desarrollo sostenible y equilibrado. Y ello solo se logra si se educa en la experiencia compartida y cooperativa en un marco de convivencia y ciberconvivencia que permita experimentar con normas comunes y resolver pacíficamente los conflictos. La investigación sobre el acoso y el ciberacoso nos ha llevado, en la última década, a profundizar en la complejidad de la competencia social y moral necesaria para que la convivencia y la ciberconvivencia se conviertan en ecosistemas de solidaridad y pro-socialidad (Romera, Bravo, Ortega-Ruiz \& Veenstra, 2019).

La EDS debe buscar que niños y niñas, adolescentes y jóvenes sepan construirse una mente prosocial que incluya la pasión por los otros, al tiempo que la suficiente competencia e inteligencia social para controlar que las reglas comunes sean democráticas y la violencia salga de las redes sociales compartidas. Pero para ello, la sociedad y la cultura, que son los referentes éticos, deben aportar, recíprocamente, un clima que no amenace dejarnos sin oxígeno para respirar, sin agricultura y ganadería que nos aporte el alimento, un sistema sanitario que garantice atención en la enfermedad y la epidemia. El camino civilizatorio apunta a un cierto altruismo universal, que garantice la no destrucción del Planeta. Si la educación quiere tener un papel en la mejora del futuro, deberá tener 
como programa mínimo la EDS, pero sin olvidar que el criterio prosocial se construye en convivencia y ciberconvivencia.

\section{Referencias}

Álvarez Munárriz L. (2001). Familia y clonación. Claves de la Razón Práctica, 109, 16-21. Apple, M. (1991). Ideología y Currículo. Madrid: Akal

Cañal, P. y Porlán, R. (1987). Investigando la realidad próxima: un modelo didáctico alternativo. Enseñanza de las Ciencias, 5(2), 89-96.

Castells, M. (1996). La sociedad red: una visión global. Madrid: Alianza Editorial.

Del Rey, R., Casas, J. A. y Ortega-Ruíz, R. (2017). Desarrollo y validación de la Escala de Convivencia Escolar (ECE). Universitas Psychologica, 16(1), 1-11. http://dx.doi.org/10.11144/Javeriana.upsy16-1.dvec

García-Pérez, F. F. (2016). Investigación en la Escuela: la construcción de una identidad. Conversación con Pedro Cañal, Eduardo García y Rafael Porlán. Investigación en la Escuela, 88, 1-21. Disponible en: http://investigacionenlaescuela.es/articulos/R88/R88-1.pdf

Giddens, A. (1991). Manual de Sociología. Madrid: Alianza Editorial

Gómez-Ortiz, O., Romera, E. M. \& Ortega-Ruíz, R. (2016). Parenting styles and bullying. The mediating role of parental psychological aggression and physical punishment. Child Abuse \& Neglect, 51, 132-143. http://dx.doi.org/10.1016/j.chiabu.2015.10.025

Habermas, J. (2001). El valle de lágrimas de la globalización. Claves de la Razón Práctica, 109, 4-10.

Held, D. \& McGrew, A. G. (Eds.) (2000). The Global Transformations Reader. Cambridge: Polity Press.

Laboratorio de Estudios sobre Convivencia y Prevención de la Violencia. Universidad de Córdoba. www.laecovi.com

Morin, E. (2004). La epistemología de la complejidad. Gaceta de Antropología, 20, artículo 02. http://hdl.handle.net/10481/7253

Ortega-Ruíz, R y Zych, I. (2016). La ciberconducta y la psicología educativa: retos y riesgos. Psicología Educativa, 22, 1-4. www.elsevier.es/psed

Ortega-Ruíz, R. (2005). Psicología de la Enseñanza y Desarrollo de Personas y Comunidades. México: Fondo de Cultura Económica.

Ortega-Ruíz, R. (2009). La educación y los derechos humanos ante los desafíos de la convivencia. En J. A. Caride (Coord.), Los derechos humanos en la educación y la cultura (pp. 137-155). Argentina: Homo Sapiens.

Ortega-Ruíz, R. (2010). Agresividad Injustificada, bullying y violencia escolar. Madrid: Alianza Editorial.

Ortega-Ruíz, R. (Coord.) (2015). Convivencia y ciberconvivencia. Un modelo educativo para la prevención del acoso y el ciberacoso escolar. Madrid: Antonio Machado Libros.

Ortega-Ruíz, R. y Martín Ortega, O. (2004). Convivencia: aspectos conceptuales, sociales y educativos. En R. Ortega y R. del Rey (Coord.), Construir la Convivencia (pp. 9-26). Barcelona: EDEBE.

Ortega-Ruíz, R., Del Rey, R. y Casas, J. A. (2015). Nuevos riesgos de la convivencia escolar: el cyberbullying. En R. Ortega-Ruiz (Coord.), Convivencia y ciberconvivencia. Un modelo educativo para la prevención del acoso y el ciberacoso escolar (pp. 99-116). Madrid: Antonio Machado Libros.

Romera, E. M., Bravo, A., Ortega-Ruíz, R. \& Veenstra, R. (2019). Diferences in perceived popularity and social preference between bullying roles and class norms. Plos ONE, 14(10): e0223499. https://doi.org/10.1371/journal.pone.0223499

Spring, J. (2001). Globalization and Educational Rights. London: Laurence Earlbaum Associates.

Trivers, R. L. (1971). The evolution of reciprocal altruism. Quarterly Review of Biology, 46, 35-57. 


\section{Información sobre la autora}

Autora: Rosario Ortega Ruíz

Institución: Universidad de Córdoba. Investigadora Principal de Laboratorio de Estudios sobre Convivencia y Prevención de la Violencia (www.laecovi.com)

Email: ortegaruiz@uco.es

ORCID: https://orcid.org/0000-0003-2110-6931 


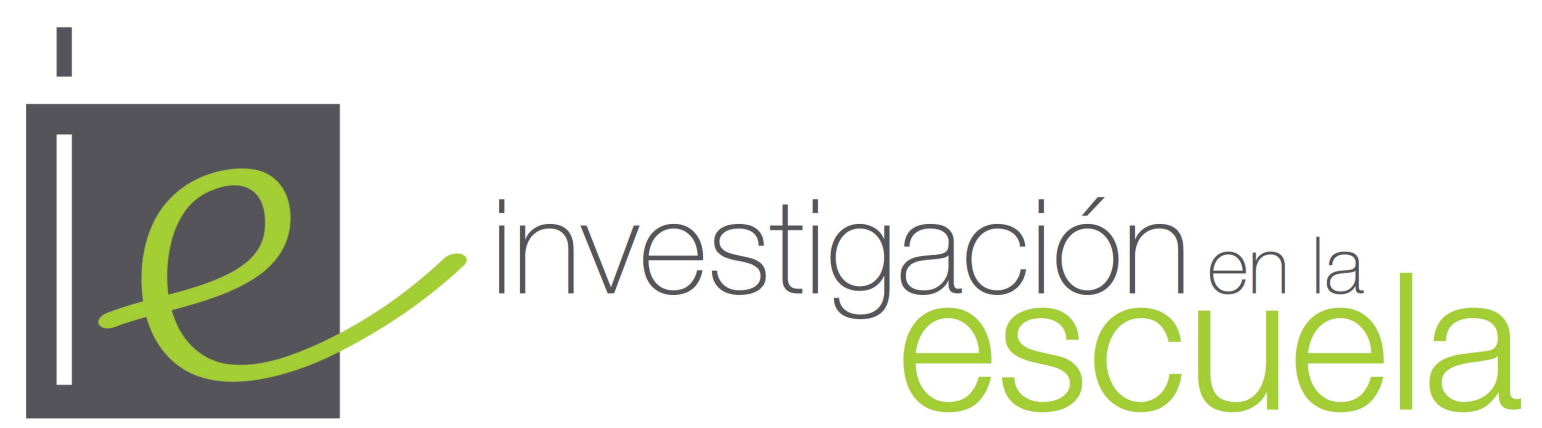

Revista académica evaluada por pares y de acceso abierto

Número 100

30 de abril de 2020

ISSN 2443-9991

\section{(c) (i) (2)}

EY SA Esta obra está bajo una licencia Creative Commons. Los/as lectores/as pueden compartir, copiar y redistribuir el material en cualquier medio o formato, así como adaptar, remezclar, transformar y construir a partir del material para cualquier propósito, incluso comercialmente. Para ello, deben de hacerlo bajo los siguientes términos: dando crédito de forma adecuada, brindando un enlace a la licencia e indicando si se han realizado cambios. Si se remezcla, transforma o crea a partir del material, debe distribuir su contribución bajo la misma licencia del original.

Más detalles de la licencia de CreativeCommons se encuentran en https://creativecommons.org/licenses/by-sa/4.0/deed.es

Cualquier otro uso debe ser aprobado en conjunto por el autor/es, o Investigación en la Escuela.

ư investigacion-en-la-escuela

Por errores y sugerencias contacte a investigacionenlaescuela@us.es

La revista Investigación en la Escuela desde su origen en 1987 hasta su no 87 (2015) fue editada por Díada Editora. 
